\title{
Graded Modalities and Resource Bisimulation
}

\author{
Flavio Corradini ${ }^{1}$, Rocco De Nicola ${ }^{2}$, and Anna Labella ${ }^{3}$ \\ 1 Dipartimento di Matematica Pura ed Applicata, Università dell'Aquila \\ flavio@univaq.it \\ 2 Dipartimento di Sistemi e Informatica, Università di Firenze \\ denicola@dsi.unifi.it \\ 3 Dipartimento di Scienze dell'Informazione, Università di Roma "La Sapienza" \\ labella@dsi.uniroma1.it
}

\begin{abstract}
The logical characterization of the strong and the weak (ignoring silent actions) versions of resource bisimulation are studied. The temporal logics we introduce are variants of Hennessy-Milner Logics that use graded modalities instead of the classical box and diamond operators. The considered strong bisimulation induces an equivalence that, when applied to labelled transition systems, permits identifying all and only those systems that give rise to isomorphic unfoldings. Strong resource bisimulation has been used to provide nondeterministic interpretation of finite regular expressions and new axiomatizations for them. Here we generalize this result to its weak variant.
\end{abstract}

\section{Introduction}

Modal and temporal logics have been proved useful formalisms for specifying and verifying properties of concurrent systems (see e.g. [19]), and different tools have been developed to support such activities [8,7]. However, to date, there is no general agreement on the type of logic to be used. Since a logic naturally gives rise to equivalences (two systems are equivalent if they satisfy the same formulae) often, for a better understanding and evaluation, the proposed logics have been contrasted with behavioural equivalences. The interested reader is referred to $[10,17]$ for comparative presentations of many such equivalences.

Establishing a direct correspondence between a logic and a behavioural equivalence provides additional confidence in both approaches. A well-known result relating behavioural and logical semantics is that reported in [15]; there, a modal logic, now known as Hennessy-Milner Logic $H M L$, is defined which, when interpreted over (arc-) labelled transition systems with and without silent actions, is proved to be in full agreement with two equivalences called strong and weak observational equivalence. Other correspondences have been established in [2] where two equivalences over Kripke structures (node-labelled transition systems) are related to two variants of $C T L^{*}$ [13], and in [12] where three different logical characterizations are provided for another variant of bisimulation called branching bisimulation.

In this paper, we study the logical characterization of yet another variant of bisimulation that we call resource bisimulation [6]. This bisimulation takes

C. Pandu Rangan, V. Raman, R. Ramanujam (Eds.): FSTTCS'99, LNCS 1738, pp. 381-393, 1999.

(C) Springer-Verlag Berlin Heidelberg 1999 
into account the number of choices a system has, even after it has decided the specific action to be performed. The new equivalence counts the instances of specific actions a system may perform and thus considers as different the two terms $P$ and $P+P$; the latter representing the non-deterministic composition of a system with itself. Intuitively, this can be motivated by saying that $P+P$ duplicates the resources available in $P$. This permits differentiating systems also relatively to a form of fault tolerance known as "cold redundancy": $P+P$ is more tolerant to faults than $P$, because it can take advantage of the different instances of the available resources.

Resource bisimulation enjoys several nice properties (we refer to [6] for a comprehensive account and for additional motivations). It has been shown that resource bisimulation coincides with the kernel of resource simulation (this result is new for simulation-like semantics). Moreover, it permits identifying all and only those labelled transition systems that give rise to isomorphic unfoldings. Also, resource bisimulation, when used to provide nondeterministic interpretation of finite regular expressions, leads to a behavioural semantics that is in full agreement with a tree-based denotational semantics and is characterized via a small set of axioms obtained from Salomaa's axiomatization of regular expressions [21] by removing the axioms stating idempotence of + and distributivity of - over + , see Table 1 .

Table 1. Axioms for resource bisimulation over finite regular expressions.

\begin{tabular}{|c|c|}
\hline $\begin{aligned} X+Y & =Y+X \\
(X+Y)+Z & =X+(Y+Z) \\
X+0 & =X\end{aligned}$ & $\begin{array}{l}(\mathrm{C} 1) \\
(\mathrm{C} 2) \\
(\mathrm{C} 3)\end{array}$ \\
\hline $\begin{aligned}(X \bullet Y) \bullet Z & =X \bullet(Y \bullet Z) \\
X \bullet 1 & =X \\
1 \bullet X & =X \\
X \bullet 0 & =0 \\
0 \bullet X & =0\end{aligned}$ & $\begin{array}{l}(\mathrm{S} 1) \\
(\mathrm{S} 2) \\
(\mathrm{S} 3) \\
(\mathrm{S} 4) \\
(\mathrm{S} 5)\end{array}$ \\
\hline$(X+Y) \cdot Z=(X \cdot Z)+(Y \cdot Z)$ & $(\mathrm{RD})$ \\
\hline
\end{tabular}

In this paper we continue our investigation on resurce bisimulation in two directions. First, we study a logical characterization of resource bisimulation, then, we provide a sound and complete axiomatization for its weak variant. The logic which characterizes resource bisimulation is obtained by replacing both the box and the diamond modalities of HML with the family of graded modalities [14], defined below, where \# denotes multisets cardinality.

$$
p \models\langle\mu\rangle_{n} \psi \text { if and only if } \#\left\{\left|p^{\prime}\right| p \stackrel{\mu}{\longrightarrow} p^{\prime} \text { and } p^{\prime} \models \psi\right\}=n \text {. }
$$


If we define Graded HML (GHML) to be the set of formulae generated by the grammar:

$$
\psi::=\text { True }\left|\psi_{1} \wedge \psi_{2}\right|\langle\mu\rangle_{n} \psi \quad \text { where } \mu \in A \text { and } 0 \leq n<\infty
$$

it can be established that

$$
(\forall \psi \in G H M L, P \models \psi \Longleftrightarrow Q \models \psi) \text { if and only if } P \sim_{r} Q
$$

We shall also study the weak variant of resource bisimulation over regular expressions enriched with a distinct invisible $\tau$-action, and we shall provide also for this new equivalence both an axiomatic and a logical characterization. The complete axiomatization will be obtained by adding the axiom

$$
\alpha \cdot \tau \bullet X=\alpha \cdot X
$$

to those for (strong) resource bisimulation of Table 1. The logical characterization is obtained by providing a different (weak) interpretation of the modal operators described above.

Due to space limitation, all proofs are omitted, they are reported in the full version of the paper.

\section{Nondeterministic Expressions and Resource Bisimulation}

In this section we provide an observational account of finite nondeterministic regular expressions, by interpreting them as equivalence classes of labelled transition systems. This part has been extensively treated in [6]. The proposed equivalence relies on the same recursive pattern of bisimulation but takes into account also the number of equivalent states that are reachable from a given one.

Let $A \cup\{1\}$ be a set of actions. The set of nondeterministic finite regular expressions over $A$ is the set $\mathbf{P L}$ of terms generated by the following grammar:

$$
P:=0|1| a|P+P| P \cdot P \text { where } a \text { is in } A .
$$

We give the following interpretation to nondeterministic regular expressions. Like in [1], the term 0 , denotes the empty process. The term 1 denotes the process that does nothing and successfully terminates. The term $a$ denotes a process that executes a visible action $a$ and then successfully terminates. The operator + can be seen as describing the nondeterministic composition of agents. The operator • models sequential composition.

Definition 1. A labelled transition system is a triple $\langle Z, L, T\rangle$ where $Z$ is a set of states, $L$ is a set of labels and $T=\{\stackrel{n}{\longrightarrow} \subseteq Z \times Z \mid n \in L\}$ is a transition relation. 
Table 2. Active predicate.

\begin{tabular}{l}
$\operatorname{active}(1)$ \\
$\operatorname{active}(a)$ \\
$\operatorname{active}(P) \vee \operatorname{active}(Q) \Longrightarrow \operatorname{active}(P+Q)$ \\
$\operatorname{active}(P) \wedge \operatorname{active}(Q) \Longrightarrow \operatorname{active}(P \bullet Q)$ \\
\hline
\end{tabular}

Table 3. Operational Semantics for PL.

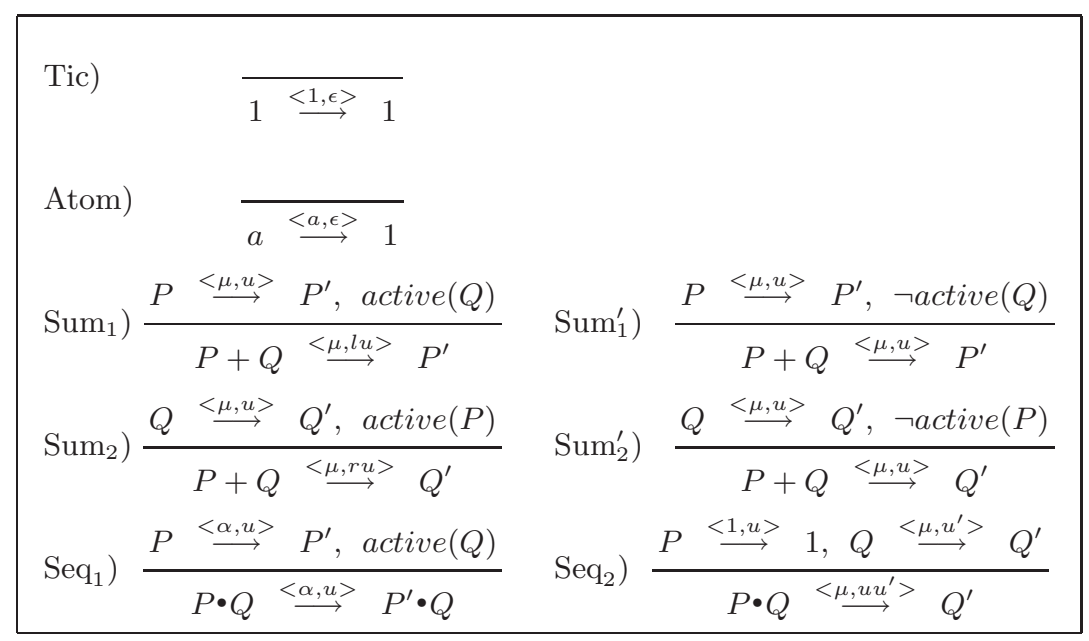

In our case, states are terms of $\mathbf{P L}$ and labels are pairs $<\mu, u>$ with $\mu \in A \cup\{1\}$ and $u$ a word, called choice sequence, in the free monoid generated by $\{l, r\}$. The transition relation relies on the "active" predicate defined in Table 2 and is defined in Table 3. There, and in the rest of the paper, we write $z \stackrel{n}{\longrightarrow} z^{\prime}$ instead of $<z, z^{\prime}>\in \stackrel{n}{\longrightarrow}$.

We have two kinds of transitions:

- $P \stackrel{<a, u>}{\longrightarrow} P^{\prime}: \quad P$ performs an action $a$, possibly preceded by 1-actions with choice sequence $u$.

- $P \stackrel{<1, u>}{\longrightarrow}$ 1: $P$ performs 1 -actions to reach process 1 with choice sequence $u$.

These transitions are atomic, which means that they cannot be interrupted and keep no track of intermediate states. In both cases, $u$ is used to keep information about the possible nondeterministic structure of $P$, and will permit distinguishing those transitions of $P$ whose action label and target state have the 
same name but are the result of different choices. Thus for $a+a$, it is possible to record that it can perform two different $a$ actions: $a+a \stackrel{<a, l>}{\longrightarrow} 1$ and $a+a \stackrel{<a, r>}{\longrightarrow} 1$; without the $l$ and $r$ labels, we would have only the $a+a \stackrel{a}{\longrightarrow} 1$ transition.

The predicate active over $\mathbf{P L}$ processes that is used in $\mathrm{Seq}_{1}$ allows us to detect empty processes and to avoid performing actions leading to deadlocked states.

The rules of Table 3 should be self-explanatory. We only comment on those for + and $\bullet$.

The rule for $P+Q$ says that if $P$ can perform $<\mu, u>$ to become $P^{\prime}$, and $Q$ is not deadlocked, then $P+Q$ can perform $\left\langle\mu, l u>\right.$ to become $P^{\prime}$ where $l$ records that action $\mu$ has been performed by the left alternative. If $Q$ is deadlocked, then, no track of the choice is kept in the label. The right alternative is dealt with symmetrically.

Seq $_{1}$ ) mimics sequential composition of $P$ and $Q$; it states that if $P$ can perform $<\mu, u>$ then $P \bullet Q$ can evolve with the same label to $P^{\prime} \bullet Q$. The premise active $(Q)$ of the inference rule ensures that $Q$ can successfully terminate.

In order to abstract from choice sequences while keeping information about the alternatives a process has for performing a specific action, we introduce a new transition relation that associates to every pair $\langle P \in \mathbf{P L}, \mu \in A c t \cup\{1\}>$, a multiset $M$, representing all processes that are target of different $\langle\mu, u\rangle$ transitions from $P$. The new transition relation is defined as the relation that satisfies:

$$
P \stackrel{\mu}{\longrightarrow}\left\{P^{\prime} \mid \exists u . P \stackrel{<\mu, u>}{\longrightarrow} P^{\prime}\right\}
$$

Thus, for example, we have:

$$
\begin{aligned}
- & a+a \stackrel{a}{\longrightarrow}\{1,1\} \text { because }-a+a \stackrel{<a, l>}{\longrightarrow} 1 \text { and }-a+a \stackrel{<a, r>}{\longrightarrow} 1, \\
- & (1+1) \cdot(a+a) \stackrel{a}{\longrightarrow}\{1,1,1,1\} \text { because } \\
& -(1+1) \cdot(a+a) \stackrel{<a, l l>}{\longrightarrow} 1,-(1+1) \cdot(a+a) \stackrel{<a, l r>}{\longrightarrow} 1, \\
& -(1+1) \cdot(a+a) \stackrel{<a, r l>}{\longrightarrow} 1-(1+1) \cdot(a+a) \stackrel{<a, r r>}{\longrightarrow} 1 .
\end{aligned}
$$

We shall now introduce the bimulation-based relations that identifies only those systems that have exactly the same behaviour and differ only for their syntactic structure. This equivalence relation, called resource bisimulation and introduced in [6], relates only those terms whose unfolding, via the operational semantics, gives rise to isomorphic labelled trees. The transition relation $\stackrel{\mu}{\longrightarrow}$, introduced above, is the basis for defining resource bisimulation.

\section{Definition 2. (Resource Bisimulation)}

a. A relation $\Re \subseteq \mathbf{P L} \times \mathbf{P L}$ is a $r$-bisimulation if for each $<P, Q\rangle \in \Re$, for each $\mu \in A \cup\{1\}:$

i. $P \stackrel{\mu}{\longrightarrow} M$ implies $Q \stackrel{\mu}{\longrightarrow} M^{\prime}$ and $\exists f$ injective: $M \rightarrow M^{\prime}$, such that $\forall P^{\prime} \in$ $M,<P^{\prime}, f\left(P^{\prime}\right)>\in \Re$;

ii. $Q \stackrel{\mu}{\longrightarrow} M^{\prime}$ implies $P \stackrel{\mu}{\longrightarrow} M$ and $\exists g$ injective: $M^{\prime} \rightarrow M$, such that $\forall Q^{\prime} \in M^{\prime},<Q^{\prime}, g\left(Q^{\prime}\right)>\in \Re$ 
b. $P$ and $Q$ are $r$-bisimilar $\left(P \sim_{r} Q\right)$, if there exists a $r$-bisimulation $\Re$ containing $\langle P, Q\rangle$.

The above definitions should be self explanatory. We just remark that the injection $f: M \rightarrow M^{\prime}$ is used to ensure that different (indexed) processes in $M$ are simulated by different (indexed) processes in $M^{\prime}{ }^{1}$. Thus r-bisimilarity requires the cardinality of $M$ be less or equal to the cardinality of $M^{\prime}$.

Since the multisets we are dealing with are finite, conditions i) and ii) of Definition 2 can be summarized as follows: $P \stackrel{\mu}{\longrightarrow} M$ implies $Q \stackrel{\mu}{\longrightarrow} M^{\prime}$ and there exists a bijective $f: M \rightarrow M^{\prime}$, s.t. for all $P^{\prime} \in M,<P^{\prime}, f\left(P^{\prime}\right)>\in \Re$.

With standard techniques it is possible to show that $\sim_{r}$ is an equivalence relation and it is preserved by nondeterministic composition and sequential composition. It is not difficult to check that $a \chi_{r} a+a, a+b \sim_{r} b+a$ and $(1+1) \cdot a \sim_{r} a+a$.

\section{A Logical Characterization of Resource Bisimulation}

In this section, we provide a positive logic for resource bisimulation. In [15], a modal logic, now known as Hennessy-Milner Logic (HML), is defined which, when interpreted over labelled transition systems with (or without) silent actions, is proved to be in full agreement with weak (or strong) observational equivalence.

Our logic is obtained from HML by eliminating the false predicate and by replacing both the box and the diamond modalities (or, alternatively, both the box and the $\neg$ modality) with a family of so called graded modalities [14] of the form $\langle\mu\rangle_{n} \varphi$ where $0 \leq n<\infty$. Intuitively, a process $P$ satisfies the formula $\langle\mu\rangle_{n} \varphi$ if $P$ has exactly $n \mu$-derivatives satisfying formula $\varphi$.

Let Graded HML (GHML) be the set of formulae generated by the following grammar:

$$
\varphi::=t t|\varphi \wedge \varphi|\langle\mu\rangle_{n} \varphi \quad \text { where } \mu \in A \cup\{1\} \text { and } 0 \leq n<\infty .
$$

The satisfaction relation $\models$ for the logic over GHML formulae is given by:

$$
\begin{array}{ll}
P=t t & \text { for any } P \\
P=\varphi_{1} \wedge \varphi_{2} & \text { iff } P \models \varphi_{1} \text { and } P \models \varphi_{2} \\
P \models\langle\mu\rangle_{n} \varphi & \text { iff } \#\left(\left\{\left|P^{\prime}\right| \exists u . P \stackrel{<\mu, u>}{\longrightarrow} P^{\prime} \mid\right\} \cap\left\{\left|P^{\prime}\right| P^{\prime} \models \varphi \mid\right\}\right)=n
\end{array}
$$

We shall let $\Re_{L}$ denote the binary relation over $P L$ processes that satisfy the same set of GHML formulae:

$$
\Re_{L}=\{(P, Q) \mid \forall \varphi \in \text { GHML }, P \models \varphi \Longleftrightarrow Q \models \varphi\}
$$

and will show that $\Re_{L}$ is a resource bisimulation.

\footnotetext{
${ }^{1}$ Since a multiset can be seen as a set of indexed elements, an injection between multisets can be seen as an ordinary injection between sets.
} 
Indeed, we can prove that the equivalence induced by GHML formulae coincides with resource equivalence. The proof that if $P \sim_{r} Q$ then, for all $\varphi \in$ GHML, it holds that $(P \mid \varphi$ iff $Q \models \varphi)$ is standard and follows by induction on the syntactic structure of formulae.

Proposition 1. Let $P, Q$ be PL processes. If $P \sim_{r} Q$ then $(\forall \varphi \in$ GHML , $P \models \varphi \Longleftrightarrow Q \models \varphi$ ).

The proof of the reverse implication, namely, the proof that any two processes satisfying the same set of GHML formulae are weak resource equivalent, requires a more sophisticated proof technique. It needs to be shown that, if $\Re_{L}$ was not a weak resource bisimulation, then, there would exist $(P, Q) \in \Re_{L}$ such that for some $\mu \in A$ ct, $P \stackrel{\mu}{\longrightarrow} M$ implies $Q \stackrel{\mu}{\longrightarrow} M^{\prime}$ and for all bijective $f_{i}: M \rightarrow M^{\prime}$, there would exist $P_{i} \in M$ such that $\left(P_{i}, f_{i}\left(P_{i}\right)\right) \notin \Re_{L}$. This implies that there exists a formula $\bar{\varphi} \in$ GHML such that $P_{i} \models \bar{\varphi}$ but $f\left(P_{i}\right) \not \bar{\varphi}$.

We can prove that, given a multiset of processes, we can find a formula characterizing each of its bisimulation classes, in the sense that every element in a class satisfies the characteristic formula of the class and does not satisfies any formula characterizing any other class. In this way, from the hypothesis that $P \sim_{r} Q$ does not hold, we can obtain a formula satisfied by one of the original processes, but not by the other one.

Given a multiset $M$ and GHML formulae $\varphi_{1}, \varphi_{2}, \ldots, \varphi_{n}$, let $M_{i}^{\varphi_{i}}$ be the subset of $M$ that satisfies $\varphi_{i}$ :

$$
M_{i}^{\varphi_{i}}=\left\{\left|P^{\prime} \in M\right| P^{\prime} \models \varphi_{i} \mid\right\} \quad i \in[1 . . n]
$$

If $\left\{M_{1}^{\varphi_{1}}, M_{2}^{\varphi_{2}}, \ldots, M_{n}^{\varphi_{n}}\right\}$ is a partition of $M$, then we shall write $M=M_{1}^{\varphi_{1}} \uplus$ $M_{2}^{\varphi_{2}} \uplus \ldots \uplus M_{n}^{\varphi_{n}}$.

Lemma 1. Given a finite multiset $M$ and a partition $M_{1} \uplus M_{2} \uplus \ldots \uplus M_{n}$ of $M$ satisfying the property that two elements of the same class satisfy the same formulae and two elements in two different classes behave differently for at least one formula, then, for every class, there is a formula (the characteristic formula) satisfied by all the elements of that class and by none of any other class. Therefore we can write $M=M_{1}^{\xi_{1}} \uplus M_{2}^{\xi_{2}} \uplus \ldots \uplus M_{n}^{\xi_{n}}$, where $\xi_{1}, \ldots, \xi_{n}$ are such that each $P \in M_{i}^{\xi_{i}}$ satisfies $\xi_{i},\left(P \models \xi_{i}\right)$, while each $Q \in M_{j}^{\xi_{j}}, j \neq i$, does not satisfy $\xi_{i}$, $\left(Q \not \models \xi_{i}\right)$.

The coincidence between resource bisimulation and the equivalence induced by the GHML formulae immediately follows from the lemma above.

Proposition 2. Let $P, Q$ be $\mathbf{P L}$ processes. If $(\forall \varphi \in$ GHML,$P \models \varphi \Longleftrightarrow Q \models \varphi)$ then $P \sim_{r} Q$. 


\section{Weak Resource Bisimulation}

This section is devoted to giving expressions in presence of invisible actions.

Let $A \cup\{1\}$ be a set of visible actions and $\tau \notin A \cup\{1\}$ be the invisible action. We use $\mu, \gamma, \ldots, \mu^{\prime}, \gamma^{\prime}, \ldots$ to range over by $A \cup\{1\} \cup\{\tau\}, \alpha, \beta, \ldots, \alpha^{\prime}, \beta^{\prime}, \ldots$ to range over by $A \cup\{\tau\}$ and $a, b, \ldots, a^{\prime}, b^{\prime}, \ldots$ to range over by $A$.

The set of nondeterministic regular expressions over $A \cup\{\tau\}$ is the set of terms generated by the following grammar:

$$
P::=0|1| a|\tau| P+P \mid P \cdot P \text { where } a \text { is in } A .
$$

We will refer to the set of terms above as $\mathbf{P L}$ as well. We extend the interpretation given for the $\tau$-less case as follows: $\tau$ denotes a process which can internally evolve and then successfully terminates. For those familiar with the operational semantic of process algebras, we would like to remark that 1-actions do not play the same role of invisible $\tau$ - actions. They simply stand for successful terminated processes.

To deal with the new actions, we extend the transition relation of Table 3 by adding the rule:

$$
\text { Tau) } \frac{}{\tau \stackrel{<\tau, \epsilon>}{\longrightarrow} 1}
$$

It relies on the predicate active defined in Table 2 extended with the condition below, that is used to detect empty processes

$$
\operatorname{active}(\tau) \text {. }
$$

We have now three kinds of transitions:

- $P \stackrel{<a, u>}{\longrightarrow} P^{\prime}: P$ performs an action $a$, possibly preceded by 1-actions, with choice sequence $u$.

- $P \stackrel{<1, u>}{\longrightarrow}$ 1: $P$ performs 1-actions to reach process 1 with choice sequence $u$.

- $P \stackrel{<\tau, u>}{\longrightarrow} P^{\prime}: P$ performs an action $\tau$, possibly preceded by 1-actions, with choice sequence $u$.

These transitions are atomic; they cannot be interrupted and, moreover, leave no track of intermediate states. In both cases, $u$ is used to keep information about the possible nondeterministic structure of $P$, and will permit distinguishing those transitions of $P$ with identical action label and target state.

Starting from elementary transitions, weak transitions can be defined. They can be invisible or visible. Weak invisible transitions denote sequences of $\tau$ transitions (possibly interleaved by 1's) that lead to branching nodes, while weak visible transitions denote the execution of visible actions (possibly) followed or preceded by invisible moves. As usual, we have also terminating moves, i.e., sequences of 1-actions leading to successful termination of a process; see Table 4 for their formal definitions. In order to be able to give full account of the differ- 
Table 4. Weak Transitions for PL.

\begin{tabular}{|c}
$\left.\mathrm{WT}_{1}\right) \frac{P_{1} \stackrel{<\mu, u>}{\longrightarrow} P_{2}}{P_{1} \stackrel{<\mu, u>}{\Longrightarrow} P_{2}}$ \\
$\left.\mathrm{WT}_{2}\right) \frac{P_{1} \stackrel{<\tau, u_{1}>}{\longrightarrow} P_{2} \text { and } P_{2} \stackrel{<\alpha, u_{2}>}{\longrightarrow} P_{3}}{P_{1} \stackrel{<\alpha, u_{1} u_{2}>}{\longrightarrow} P_{3}}$ \\
$\left.\mathrm{WT}_{3}\right) \frac{P_{1} \stackrel{<\stackrel{\alpha, u_{1}}{\longrightarrow}}{\longrightarrow} P_{2} \text { and } P_{2} \stackrel{<\tau, u_{2}>}{\longrightarrow} P_{3}}{P_{1} \stackrel{<\alpha, u_{1} u_{2}>}{\longrightarrow} P_{3}}$
\end{tabular}

ent alternatives a process has when determining the specific action to perform, we introduce a transition relation that associates a multiset $M$, to every pair $P \in \mathbf{P L}, \mu \in A c t \cup\{1\} \cup\{\tau\} . M$ represents all processes that reacheable via (initial) weak $\langle\mu, u>$-transitions by $P$. Since we are interested in the branching structure of processes and in detecting their actual choice points, we remove from $M$ all those processes which can perform a $\tau$ actions in a purely deterministic fashion. That is, we remove those target processes which can perform an initial $\tau$-transition "without choice". This new transition relation is defined as the least relation such that:

$$
P \stackrel{\mu}{\Longrightarrow}\left\{\left|P^{\prime}\right| \exists u . P \stackrel{<\mu, u>}{\Longrightarrow} P^{\prime} \mid\right\}-\left\{\left|P^{\prime}\right| P^{\prime} \stackrel{<\tau, v>}{\Longrightarrow} \text { with } v=\epsilon \mid\right\} .
$$

The transition relation $\stackrel{\mu}{\Longrightarrow}$ is the basis for defining resource equivalence.

\section{Definition 3. (Weak Resource Bisimulation)}

1. A relation $\Re \subseteq \mathbf{P L} \times \mathbf{P L}$ is a weak resource bisimulation if for each $\langle P, Q\rangle \in$ $\Re$, for each $\mu \in A \cup\{1\} \cup\{\tau\}$ :

(i) $P \stackrel{\mu}{\Longrightarrow} M$ implies $Q \stackrel{\mu}{\Longrightarrow} M^{\prime}$ and there exists an injective $f: M \rightarrow M^{\prime}$, such that for all $P^{\prime} \in M,<P^{\prime}, f\left(P^{\prime}\right)>\in \Re$;

(ii) $Q \stackrel{\mu}{\Longrightarrow} M^{\prime}$ implies $P \stackrel{\mu}{\Longrightarrow} M$ and there exists an injective $g: M^{\prime} \rightarrow M$, such that for all $Q^{\prime} \in M^{\prime},<g\left(Q^{\prime}\right), Q^{\prime}>\in \Re$.

2. $P$ and $Q$ are weak resource equivalent $\left(P \approx_{r} Q\right)$, if there exists a weak r-bisimulation $\Re$ containing $<P, Q>$.

Remark 1. An immediate difference between weak resource equivalence and the standard observational equivalence, see e.g. [18], is that we do not consider "empty" $\tau$ - moves, i.e., moves of the form $P \stackrel{<\epsilon, \epsilon>}{\Longrightarrow} P$; we require at least one $\tau$ to be performed. In our framework, a process cannot idle to match a transition of another process which performs invisible actions. 
Below, we provide a number of examples that should give an idea of the discriminating power of weak resource equivalence:

- Processes $\tau \bullet \tau$ and $\tau$ are related, while $\tau+\tau$ and $\tau$ are taken apart. The reason for the latter differentiation is similar to that behind $1+1 \not \nsim_{r} 1$. Indeed $(\tau+\tau) \bullet a$ is equal to $\tau \bullet a+\tau \bullet a$ which has to be different (in this counting setting) from $\tau \bullet a$.

- Processes $a \bullet \tau$ and $a$ are related because the $\tau$ following action $a$ is not relevant from the branching point of view.

- $\tau \bullet a$ and $a$ are instead distinguished because the $\tau$ action preceding the $a$ in the former process can influence choices when embedded in a non-deterministic context.

- Processes $\tau$ and 1 are not equivalent, again because a $\tau$ action can be ignored only after a visible move.

- Processes $(\tau+1) \bullet a$ and $a+\tau \bullet a$ are weak resource bisimilar.

- Processes $\tau(\tau+0)$ and $\tau \bullet \tau$ are weak resource bisimilar

The following proposition states a congruence result for weak resource bisimulation. We can prove that our equivalence is actually preserved by all PL operators; noticeably it is preserved by + . This is another interesting property of our equivalence notion; weak equivalences are usually not preserved by + and additional work is needed to isolate the coarsest congruence contained in them.

Proposition 3. Weak resource equivalence is preserved by all PL operators.

Let us consider now the simulation relation, denoted by $\preceq_{r}$ and called weak resource simulation, obtained by removing item 1.(ii) from Definition 3. It can be can shown that (like for strong resource bisimulation) the kernel of $\preceq_{r}$, coincide with weak resource equivalence.

Proposition 4. Let $\preceq_{r}$ be the preorder obtained by considering one of the two items in the definition of $\approx_{r}$, and let $P$ and $Q$, be two processes. Then, $P \approx_{r} Q$ iff $P \preceq_{r} Q$ and $Q \preceq_{r} P$.

The logical characterization of resource equivalence can be easily extended to the weak case. It is sufficient to extend the alphabet and to introduce a $\tau$ modality. Then, within the actual definition of the satisfaction relation

$-P \stackrel{<\mu, u>}{\longrightarrow} P^{\prime}$ has to be replaced by $P \stackrel{<\mu, u>}{\longrightarrow} P^{\prime}$;

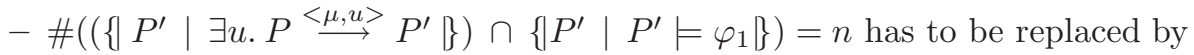
$\#\left(\left(\left\{\left|P^{\prime}\right| \exists u . P \stackrel{<\mu, u>}{\Longrightarrow} P^{\prime} \mid\right\}-\left\{\left|P^{\prime}\right| P^{\prime} \stackrel{<\tau, v>}{\Longrightarrow}\right.\right.\right.$ with $\left.\left.\left.v=\epsilon \mid\right\}\right) \cap\left\{\left|P^{\prime}\right| P^{\prime} \models \varphi_{1} \mid\right\}\right)=n$.

- $P \sim_{r} Q$ has to be replaced by $P \approx_{r} Q$.

Proposition 5. The equivalence induced by extended weak GHML formulae coincides with weak resource equivalence. 
Table 5. The $\tau$-law for EPL.

$$
\alpha \cdot \tau \bullet X=\alpha \cdot X
$$

A sound and complete axiomatization of weak resource equivalence over $\mathbf{P L}$ processes can also be provided. We can prove that the new weak equivalence is fully characterized by the axiom of Table 5 (please remember that now $\alpha \in$ $A \cup\{\tau\}$ ) together with the set of axioms of Table 1 (which soundly and completely axiomatize strong resource equivalence [6]).

Proposition 6. The axiom of Table 5 together with the set of axioms of Table 1 soundly and completely axiomatize weak resource bisimulation over PL.

Remark 2. Consider the axiom of Table 5 and replace action $\alpha$ with $\mu$. The resulting axiom, $\mu \bullet \tau \bullet X=\mu \bullet X$, is not sound. Indeed, by letting $\mu=1$ and $X=1$ we would have that $1 \bullet \tau \bullet 1$ and $1 \bullet 1$ are related by the equational theory, while they are not weak resource equivalent as remarked above. Therefore the axiom $X \cdot \tau \bullet Y=X \cdot Y$ is not sound.

\section{Conclusions}

We have introduced graded modalities and used them to provide a logical characterization of the strong and weak versions of resource bisimulation, an equivalence which discriminates processes according to the number of different computation they can perform to reach specific states. As a result, resource bisimulation identifies all and only those labelled transition systems that give rise to isomorphic unfoldings. In the case of the weak variant this isomorphism is guaranteed up to ignoring the invisible $\tau$-actions. We have also extended the complete axiomatization of strong resource bisimulation of [6] to the weak variant of the equivalence.

The results that we have obtained for regular espressions can easily be extended to full-fledged process algebras like CCS, CSP, ACP or variants thereof that are equipped with a structural operational semantics, if care is taken to properly model the choice operators.

The logic we have introduced to characterize both resource and weak resource bisimulation, can easily be related with other modal logics introduced for dealing with bisimulation. In particular, referring to [9] as a comprehensive treatment, we can describe our logic as a polymodal $\aleph_{O}$ graduated logic. Also in [9], bisimulation is a $k$-counting bisimulation, that in the case $k=\aleph_{O}$ coincide with our resource bisimulation. On the other hand the two points of view are completely different and, in some sense, complementary. There, one was interested in the largest logic (the more expressive one) invariant under bisimulation. Here, we are looking for the minimal logic that is sufficient for characterizing bisimilar 
processes. As a consequence, our logic is extremely poor in connectives (just the conjunction) as well as in atomic propositions (just $t t$ ). In this way we showed that for example negation is not necessary. We have extended also our result to the case in which a "silent" relation $\tau$ between worlds is allowed, while we have not explicitly treated infinite terms. Nonetheless it can be immediately seen, by looking to the structure of proofs, that, if we allow infinite terms corresponding to behaviours with finite branching, e.g. guarded by $\mu$ operators, all the results will still hold. This is in accordance with the fact that, in the modal logics quoted above, $\mu$ operators are introduced for positive formulae only, and our language consists of strict positive formulae.

\section{References}

1. Baeten,J.C.M., Bergstra,J.A.: Process Algebra with a Zero Object. In Proc. Concur'90, LNCS 458, pp. 83-98, 1990. 383

2. Browne,M.C., Clarke,E., Grümberg O.: Characterizing Finite Kripke Structures in Propositional Temporal Logic. Theoretical Computer Science 59(1,2), pp. 115-131, 1998. 381

3. Baeten,J., Weijland, P.: Process Algebras. Cambridge University Press, 1990.

4. Corradini,F., De Nicola,R. and Labella,A.: Fully Abstract Models for Nondeterministic Regular Expressions. In Proc. Concur'95, LNCS 962, Springer Verlag, pp. 130-144, 1995.

5. Corradini,F., De Nicola,R. and Labella,A.: A Finite Axiomatization of Non deterministic Regular Expressions. Theoretical Informatics and Applications. To appear. Available from: ftp://rap.dsi.unifi.it/pub/papers/FinAxNDRE. Abstract in FICS, Brno, 1998.

6. Corradini,F., De Nicola,R. and Labella,A.: Models for Non deterministic Regular Expressions. Journal of Computer and System Sciences. To appear. Available from: ftp://rap.dsi.unifi.it/pub/papers/NDRE. 381, 382, 383, 385, 391, 391

7. Clarke,E.M., Emerson,E.A., Sistla,A.P.: Automatic Verification of Finite State Concurrent Systems using Temporal Logic Specifications. ACM Toplas 8(2), pp. 244-263, 1986. 381

8. Cleaveland,R., Parrow,J., Steffen,B.: The Concurrency Workbench. ACM Toplas 15(1), pp. 36-72, 1993. 381

9. D'Agostino,G.: Modal Logics and non well-founded Set Theories: translation, bisimulation and interpolation. Thesis, Amsterdam, 1998. 391, 391

10. De Nicola,R.: Extensional Equivalences for Transition Systems. Acta Informatica 24, pp. 211-237, 1987.381

11. De Nicola,R., Labella,A.: Tree Morphisms and Bisimulations, Electronic Notes in TCS 18, 1998.

12. De Nicola,R., Vaandrager,F.: Three Logics for Branching Bisimulation. Journal of ACM 42(2), pp. 458-487, 1995. 381

13. Emerson,E.H., Halpern,Y.: "Sometimes" and "not never" revisited: On branching versus linear time temporal logic. Journal of ACM 42, pp. 458-487, 1995. 381

14. Fattorosi-Barnaba,M., De Caro,F.: Graded Modalities, I. Studia Logica 44, pp. 197-221, 1985. 382, 386

15. Hennessy,M., Milner,R.: Algebraic Laws for Nondeterminism and Concurrency. Journal of ACM 32, pp. 137-161, 1985. 381, 386 
16. Hoare, C.A.R.: Communicating Sequential Processes, Prentice Hall, 1989.

17. van Glabbeek,R.J.: Comparative Concurrency Semantics and Refinement of Actions. Ph.D. Thesis, Free University, Amsterdam, 1990. 381

18. Milner,R.: Communication and Concurrency, Prentice Hall, 1989. 389

19. Manna,Z., and Pnueli,A.: The Temporal Logic of Reactive and Concurrent Systems. Springer Verlag, 1992. 381

20. Park,D.: Concurrency and Automata on Infinite sequences. In Proc. GI, LNCS 104, pp. 167-183, 1981.

21. Salomaa,A.: Two Complete Axiom Systems for the Algebra of Regular Events. Journal of ACM 13, pp. 158-169, 1966. 382 